\title{
Depresión postparto en el Perú
}

\section{Postpartum depression in Peru}

La depresión postparto es un concepto cuya definición todavía no está bien establecida (1). El DSM-IV tenía el especificador "de inicio en el postparto" que se usaba para los cuadros psiquiátricos (depresión mayor, manía, episodio mixto o psicosis breve) que comenzaban durante las 4 semanas siguientes al parto (2); mientras que la CIE10 se refiere a "trastornos mentales asociados al puerperio" si estos comienzan en las 6 primeras semanas postparto (3). Sin embargo, un panel de expertos ha sugerido que el periodo que debe considerarse es el de los 3 primeros meses luego del nacimiento (1). Un estudio realizado en Edimburgo, halló que el riesgo relativo (RR) de patología psiquiátrica es especialmente alto durante los 30 primeros días postparto $(\mathrm{RR}=6)$, va disminuyendo a medida que nos vamos alejando del momento del nacimiento ( $R R=3,8$, si se considera el periodo de los 90 días postparto) y permanece elevado hasta los 2 años siguientes al parto ( $R R=1,3$ entre los 90 días y los 2 años postparto) (4).

Considerando específicamente a la depresión, los 5 meses siguientes al parto tienen mayor riesgo de presentar este trastorno y además hasta los 11 meses postparto el riesgo se mantiene elevado en comparación con el periodo de gestación (5).

Por otro lado, el DSM-5, tomando en cuenta que también la gestación es un periodo de mayor riesgo de patología psiquiátrica, considera el especificador "de inicio en el periparto" para hacer referencia a los trastornos mentales que comienzan durante el embarazo o las 4 primeras semanas postparto (6).

En el presente número de la Revista Médica Herediana, Masías y Arias (7), publican un estudio que evalúa la prevalencia de sintomatología depresiva postparto mediante la Escala de Depresión Postnatal de Edimburgo (Edinburgh Postnatal Depression Scale, EPDS) en una posta de la ciudad de Arequipa (Perú). La EPDS (8) es una escala de 10 ítems que fue diseñada con la finalidad de evaluar síntomas depresivos en el periodo postparto. La necesidad de usar un instrumento especial para el postparto en vez de las escalas usuales para depresión surge al considerar que durante el puerperio hay manifestaciones físicas que podrían elevar los puntajes de ciertos ítems de las escalas convencionales que hacen referencia a sintomatología somática, y de este modo, generar confusión.

En Lima-Perú, se realizó un estudio de validación de la EPDS (9) en el que se sugiere usar un punto de corte de $\geq$ 14; con el que se obtiene una sensibilidad de $84,21 \%$ y una especificidad de 79,47\% para el diagnóstico de depresión mayor basada en los criterios del DSM-IV. Masías y Arias, sin embargo, utilizan un punto de corte algo menor $(\geq 13)$ para calcular la prevalencia de "probable depresión postparto", que según el estudio de validación hecho en Lima tendría una sensibilidad de $89,47 \%$ y una especificidad de $72,19 \%$. Con ese punto de corte se tiene más sensibilidad pero a costa de menor especificidad y encuentran una prevalencia de $14,15 \%$. Hay que tener en cuenta que como la sensibilidad y especificidad no son $100 \%$, el porcentaje de participantes que presenta valores en la EPDS iguales o superiores al punto de corte (ya sea 13 o 14), no corresponde exactamente a la prevalencia de depresión mayor. Para poner las cifras en contexto, el porcentaje de puérperas con EPDS $\geq 13$ fue $34 \%$ en un estudio realizado en el Hospital Dos de Mayo (Lima) (10) y de 31,46\% en el estudio de validación de la EPDS en el Hospital Cayetano Heredia (Lima); y en esta última muestra se encontró una prevalencia de depresión mayor según el DSM-IV de $5,92 \%(11)$.

Considerando lo expuesto, la prevalencia real de depresión en la muestra del estudio de Masías y Arias probablemente sea bastante más baja que el 14,15\% encontrado; y aún más baja que el 5,92\% hallado en Lima. Comparativamente tenemos que la prevalencia puntual de depresión en mujeres adultas de Lima Metropolitana y el Callao es de alrededor de 3,8\% (12); asimismo, los porcentajes de depresión en mujeres con más de dos años 
Tabla 1. Variables asociadas a depresión postparto en estudios realizados en Latinoamérica (13).

- Extremos de edad: madre joven (por ejemplo, $<19$ ó 25 años) o añosa (>34 años).

- Ser soltera, separada o viuda; ausencia del padre del niño.

- Pobre apoyo o conflictos con la pareja y la familia.

- Bajo nivel socio-económico.

- Bajo grado de instrucción.

- Bajos ingresos económicos / problemas financieros.

- Poco acceso de servicios de salud.

- Trabajo excesivo.

- Pensamientos e intento de terminar la gestación.

- Mayor número de gestaciones previas o hijos (por ejemplo, 3 ó más), aunque otros estudios encuentran que el menor número de gestaciones previas se relaciona con mayor riesgo.

- Seis o más años de vida marital.

- Raza blanca o negra.

- Antecedente de aborto previo.

- Embarazo no planificado o actitud negativa hacia la gestación.

- Pobre control prenatal.

- Alguna enfermedad durante el embarazo (entre ellas, infección del tracto urinario).

- Desarrollo de várices

- Cesárea (aunque también se ha encontrado como factor de riesgo al parto vaginal).

- Hijo prematuro, con bajo peso al nacer, con talla pequeña, con riesgo de morir o que haya necesitado hospitalización.

- Suspensión o dificultades con la lactancia materna.

- Cólico infantil.

- Fumar.

- Historia familiar de problemas de salud mental.

- Diagnóstico previo de depresión o problemas emocionales en algún momento de la vida.

- Trastorno disfórico premenstrual.

- Depresión o ansiedad durante el embarazo.

- Disforia durante el último embarazo o luego de gestaciones previas.

- Blues postnatales.

- Privación de sueño.

- Trastorno obsesivo-compulsivo.

- Eventos vitales adversos.

- Enfermedad seria de persona cercana.

postparto tuvieron una tasa de depresión significativamente más alta de las que tenían menos de dos años postparto (11).

Masías y Arias hacen referencia a un porcentaje de síntomas depresivos de 45,13\% que corresponde a las participantes que tienen puntajes entre 10 y 12 en la EPDS, que si lo sumamos al 14,15\% de mujeres con "probable 
depresión" obtendremos un total de 59,28\%, valor bastante parecido al 57,63\% encontrado en el estudio de validación de la EPDS en Lima para un punto de corte de $\geq 10$. Estos porcentajes tan altos, sugieren que puntos de corte menores de 13 tienen poca utilidad práctica.

Respecto a los factores de riesgo, los estudios realizados en Latinoamérica identifican los listados en la tabla 1 (13). Las variables asociadas a depresión postparto sugieren que, en su origen, como en la depresión que ocurre en otros momentos de la vida, participan factores psicosociales y factores biológicos; estos últimos particularmente se relacionan con cambios hormonales (por ejemplo, trastorno disfórico premenstrual y blues postnatales). Masías y Arias encuentran relación entre menor grado de instrucción y más depresión; relación que ocurre en general con la depresión y la ansiedad (14) (no solo con la que se presenta en el puerperio).

En cuanto a la estructura de la EPDS, se conoce que consta de 3 factores: a) anhedonia, ítems 1 y 2; b) ansiedad, ítems 3 al 6; y c) depresión, ítems del 7 al 10 (15). Masías y Arias reportan 2 factores, el factor I corresponde a la combinación de síntomas de depresión y ansiedad; y el factor II, a los síntomas de anhedonia más el ítem 4 ("he estado ansiosa y preocupada sin una buena razón") que se refiere a sintomatología ansiosa. Una explicación por la que fue posible identificar sólo 2 factores es el poco tamaño de muestra (para análisis factorial se recomienda muestras más grandes). El estudio de validación de la EPDS realizado en Lima sugiere que 3 de los 4 ítems que tienen mayor capacidad de predecir depresión postparto evalúan síntomas ansiosos (ítems 4, 5 y 6) (9).

Para concluir podemos comentar que los estudios realizados en el Perú indican que la depresión postparto tiene: a) una prevalencia similar a la que puede encontrarse en otros momentos de la vida de la mujer; b) un componente ansioso importante; y c) factores de riesgo psicosociales y biológicos; y estos últimos relacionados a una mayor sensibilidad emocional ante cambios hormonales.

\section{Johann M. Vega-Dienstmaier ${ }^{1, a}$}

\section{REFERENCIAS BIBLIOGRÁFICAS}

1. Wisner KL, Moses-Kolko EL, Sit DKY. Postpartum depression: a disorder in search of a definition. Arch Womens Ment Health. 2010; 13:37-40.

2. American Psychiatric Association. Manual diagnóstico y estadístico de los trastornos mentales. DSM-IV. Barcelona: Masson; 1996.

3. World Health Organization. Clasificación de los trastornos mentales y del comportamiento. Con glosarios y criterios diagnósticos de investigación CIE-10: CDI-10. Madrid: Editorial Médica Panamericana SA; 2000.

4. Kendell RE, Chalmers JC, Platz C. Epidemiology of puerperal psychoses. Br J Psychiatry. 1987; 150:662673.

5. Munk-Olsen T, Laursen TM, Pedersen CB, Mors O, Mortensen PB. New parents and mental disorders: a population-based register study. JAMA. 2006; 296:2582-2589.

6. Sharma V, Mazmanian D. The DSM-5 peripartum specifier: prospects and pitfalls. Arch Womens Ment Health. 2014; 17:171-173.
7. Masías M, Arias W. Prevalencia de la depresión postparto en Arequipa, Perú. Rev Med Hered. 2018;29:226-231. DOI: https://doi.org/10.20453/ rmh.v29i4.3444

8. Cox JL, Holden JM, Sagovsky R. Detection of postnatal depression: Development of the 10-item Edinburgh Postnatal Depression Scale. $\mathrm{Br} \quad \mathrm{J}$ Psychiatry. 1987; 150:782-786.

9. Vega-Dienstmaier JM, Mazzotti G, Campos M. Validación de una versión en español de la Escala de Depresión Postnatal de Edimburgo (Validation of a Spanish version of the Edinburgh Postnatal Depression Scale). Actas Esp Psiquiatr.2002; 30:106111.

10. Campos EN. Nivel de depresión postparto en puérperas atendidas en el Hospital Nacional Dos de Mayo de Lima - Perú, 2017, Tesis de Licenciatura en Psicología. Lima: Universidad Inca Garcilaso De La Vega, Facultad de Psicología y Trabajo Social;2018.

11. Vega-Dienstmaier JM, Mazzotti G, StucchiPortocarrero S, Campos M. Prevalencia y factores de riesgo para depresión en mujeres postparto. Actas 
Esp Psiquiatr. 1999;27(5):299-303.

12. Instituto Nacional de Salud Mental. Estudio Epidemiológico de Salud Mental en Lima Metropolitana y Callao Replicación 2012: Informe General. Anales de Salud Mental. 2013; 29:1-392.

13. Vega-Dienstmaier J, Zapata-Vega MI. Postpartum depression: Latin-American perspectives. In: Rosenfield AI, editor. New Research on Postpartum Depression. 2007. pp. 29-46.
14. Bjelland I, Krokstad S, Mykletun A, Dahl AA, Tell GS, Tambs K. Does a higher educational level protect against anxiety and depression? The HUNT study. Soc Sci Med. 2008; 66:1334-1345.

15. Coates R, Ayers S, de Visser R. Factor structure of the Edinburgh Postnatal Depression Scale in a populationbased sample. Psychol Assess. 2017;29:1016-1027. 